\title{
Evidence for an intermediate-mass black hole in NGC 6624
}

\section{B. B. P. Perera ${ }^{1}$, B. W. Stappers ${ }^{1}$, A. G. Lyne ${ }^{1}$, C. G. Bassa ${ }^{2}$, I. Cognard ${ }^{3,4}$, L. Guillemot ${ }^{3,4}$, M. Kramer ${ }^{5,1}$, G. Theureau ${ }^{3,4,6}$ and G. Desvignes ${ }^{5}$}

\author{
${ }^{1}$ Jodrell Bank Centre for Astrophysics, School of Physics and Astronomy, The University of \\ Manchester, Manchester M13 9PL, UK \\ ${ }^{2}$ ASTRON, the Netherlands Institute for Radio Astronomy, Postbus 2, 7990 AA, Dwingeloo, \\ the Netherlands \\ ${ }^{3}$ Laboratoire de Physique et Chimie de l'Environnement et de l'Espace LPC2E \\ CNRS-Université d'Orléans, F-45071 Orléans, France \\ ${ }^{4}$ Station de radioastronomie de Nançay, Observatoire de CNRS/INSU, F-18330 Nançay, France \\ ${ }^{5}$ Max-Planck-Institut für Radioastronomie, Auf dem Hügel 69, D-53121 Bonn, Germany \\ ${ }^{6}$ Laboratoire Univers et Théories LUTh, Observatoire de Paris, CNRS/INSU, Université Paris \\ Diderot, 5 place Jules Janssen, F-92190 Meudon, France
}

\begin{abstract}
PSR B1820-30A is located in the globular cluster NGC 6624 and has the smallest projected distance to the centre of any globular cluster in the sky plane. We observe this millisecond pulsar over more than 25 years and obtain higher-order rotational frequency time derivative measurements through high-precision timing. Modelling these higher-order derivatives as being due to orbital motion, we find that the pulsar is in either a low-eccentricity smaller orbit with a low mass companion or a high-eccentricity larger orbit with a massive companion. The cluster mass properties and the observed properties of other nearby sources indicate that the high-eccentricity solution is more probably. This reveals that the pulsar is orbiting around an intermediate-mass black hole (IMBH) of mass $>7500 \mathrm{M}_{\odot}$ located at the cluster centre. This contribution is based on previous work published in MNRAS 471, 1258 (2017).
\end{abstract}

Keywords. black hole physics-stars: neutron-pulsars: individual: PSR B1820-30A- globular clusters: individual: NGC 6624

\section{Introduction}

Globular clusters contain large numbers of old stars (typically $10^{5}-10^{6}$ ) gravitationally bound together in regions of a few tens of light years across. Their high central stellar densities provide a likely environment to form massive black holes (BHs - Portegies Zwart et al. 2004). The dynamics of the central region are dominated by the presence of any central $\mathrm{BH}$ and the motion of stars around the $\mathrm{BH}$ can potentially be measured. Millisecond pulsars (MSPs) are stable rotators and thus, they are very sensitive to any dynamical changes caused by the presence of a central $\mathrm{BH}$.

The Milky Way globular cluster NGC 6624 is located $7.6 \pm 0.4 \mathrm{kpc}$ away from the Earth (Kuulkers et al. 2003), and $1.2 \mathrm{kpc}$ away from the Galactic centre (Harris 1996). Six radio pulsars have been discovered so far in NGC 6624: PSRs B1820-30A and B (Biggs et al. 1994), and PSRs J1823-3021C, D, E and F (Chandler 2003; Lynch et al. 2012). PSRs B1820-30B and J1823-3021C are young pulsars with a spin period of $\sim 0.4$ $\mathrm{s}$, while the others are MSPs with a spin period of $<6 \mathrm{~ms}$. PSR B1820-30A is the closest known pulsar to the centre of any globular cluster, where the projected distance from the cluster centre in the sky-plane is $\approx 0.5$ arcsec. Its rotational frequency $(f)$ is $183.82 \mathrm{~Hz}$ and its time derivative $(\dot{f})$ is $-1.14 \times 10^{-13} \mathrm{~Hz} / \mathrm{s}$ (Lynch et al. 2012). This pulsar provides 


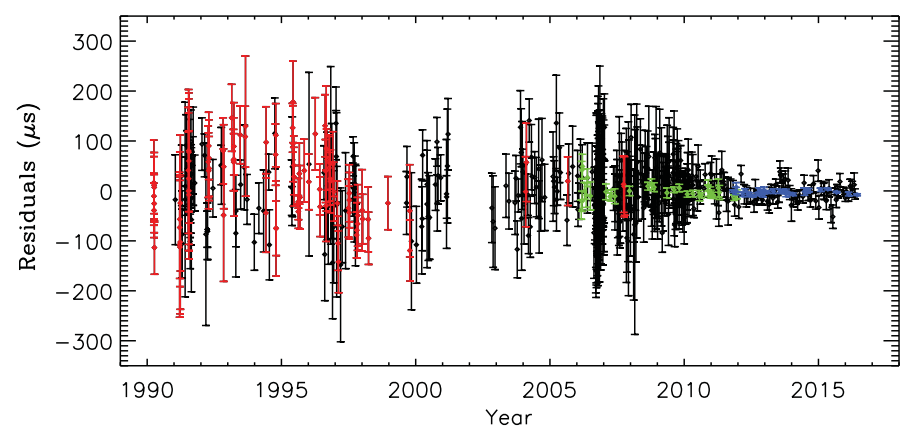

Figure 1. The timing residuals of PSR B1820-30A. The observations with different frequencies and backends are shown in different colours: LT $1400 \mathrm{MHz}$ (black), LT $600 \mathrm{MHz}$ (red), NRT BON (green), and NRT NUPPI (blue). Note that the colours can only be seen in the online version.

the highest measured $\dot{P}$ for any MSP and is several orders of magnitude greater than the typical value (see Manchester et al. 2005). It has previously been proposed that this anomalous $\dot{P}$ is not intrinsic to the pulsar and is solely induced by the dynamics due to its close location in the cluster centre (Biggs et al. 1994).

\section{Observations and timing the pulsar}

PSR B1820-30A was observed using the Lovell Telescope (LT) at the Jodrell Bank Observatory in the UK since its discovery in 1990 March. The observations were made roughly every $18 \mathrm{~d}$ over more than $25 \mathrm{yr}$, providing the largest ever data set available for this pulsar, resulting in a total of 516 epochs. In addition to the LT observations, the pulsar was observed using the Nançay Radio Telescope (NRT) in France since 2006 February, resulting in a total of 61 epochs. We folded the data of each observation in time and frequency, and then obtain the time-of-arrival (TOA) of the pulse. We then fit a standard pulsar timing model and obtain the residuals between the observed and model predicted TOAs.

We started the fit by including only $f$ and $\dot{f}$ in the model and found a large remaining structure in the residuals. We found that it is necessary to include higher order frequency derivatives to minimise these residuals, up to the fourth order (i.e. $f^{(4)}$ ), where the resultant accuracy of $f^{(4)}$ was $2.2 \sigma$. The timing residuals are shown in Figure 1 . We also fit for $f^{(5)}$ and found that its uncertainty is large, so that it provides only an upper limit (see Perera et al. (2017a), for details).

The measured second-order rotational frequency derivative is several orders of magnitude greater than that of other MSPs in general (Manchester et al. 2005). Since MSPs are stable rotators, we do not expect to see such large and higher order spin-down as we observe here. Therefore, these higher order derivatives are not likely to be intrinsic to the pulsar, rather dynamically induced due to binary motion.

\section{Determining the orbital parameters and masses}

The observed $\dot{P}$ of a globular cluster pulsar is somewhat different from its intrinsic value due to various acceleration terms. We consider all possible acceleration terms (e.g. accelerations due to differential Galactic potential, towards the Galactic plane, cluster potential, and the proper motion of the pulsar) and find that observed $\dot{P}$ is dominated by the cluster potential. This suggests that the observed $\dot{P}$ is induced by the orbital 
motion of the pulsar. Thus, we apply the model proposed in Joshi \& Rasio (1997) to the timing data to determine all possible orbital system parameters and the companion masses consistent with the higher order frequency derivatives.

We first investigate all possible orbital systems of the pulsar based on its measured higher order rotational frequency derivatives through timing. We search for solutions using the initial guesses for the orbital parameters - eccentricity $e$, the longitude of periastron $\omega$ (measured from the ascending node), the longitude of the reference epoch $\lambda$ (measured from the periastron) - across their entire space. This gives valid solutions only when $e \gtrsim 0.33$. We then use the valid orbital parameters to estimate the modelpredicted $f^{(5)}$ and then compare that with the measured $3 \sigma$ upper limit of $f^{(5)}$. This rules out all orbits where the eccentricity lies in the range $0.56-0.9$. This results in possible high-eccentricity larger orbits with massive companions and low-eccentricity smaller orbits with low-mass companions. We note that these high-eccentricity orbits are large enough to pass through the cluster centre. As described in Peuten et al. (2014), the observations of the low-mass X-ray binary (LMXB) 4U 1820-30, which is located close to PSR B1820-30A, indicate that it is orbiting around the cluster centre, and thus, we consider that all the possible smaller orbits with low-mass companions are unlikely.

We then examine the parameters of the pulsar when it is in a high-eccentricity orbit where $e \gtrsim 0.9$. We first assume that the intrinsic spin down of the pulsar is negligible and then constrain the orbital parameters and the companion masses. We calculate the model-dependent projected distance of the pulsar from the cluster centre using orbital parameters. By comparing the model-dependent projected distance with the measured projected distance (i.e. $0.016-0.042 \mathrm{pc}$ ) obtained from the cluster centre and pulsar position measurements, we constrain the lower limit of the orbital inclination to be $44^{\circ}$ (see Perera et al. 2017a, for details). Therefore, the timing measured $f^{(4)}$ and the orbital inclination range $44^{\circ}<i<90^{\circ}$ constrain the orbit size, orbital period, and the companion mass to be $(0.012-0.025) \mathrm{pc},(1.36-1.94) \mathrm{kyr}$, and $8000-37000 \mathrm{M}_{\odot}$, respectively. The companion mass results are shown in Figure 2.

In principle, the pulsar might be orbiting around the cluster centre full of stars, however, combining this mass with the periastron separation of the pulsar orbit $(9-14) \times$ $10^{-5} \mathrm{pc}$, where this range is due to the possible orbital inclinations, this leads to a stellar density of $3 \times 10^{15} \mathrm{M}_{\odot} / \mathrm{pc}^{3}$ compared with the low central density of the cluster of $\sim 10^{5} \mathrm{M}_{\odot} / \mathrm{pc}^{3}$ derived from the early optical observations (Canizares et al. 1978). Such an exceptional mass density can only be achieved if the pulsar is orbiting around an IMBH.

Furthermore, we also assume a significant intrinsic spin-down of the pulsar in the model and obtain the orbital parameters and the companion mass using the same procedure given above. We find that the companion mass is $M>7500 \mathrm{M}_{\odot}$ and again the periastron distance is small, resulting in extremely high central mass density, indicating a central IMBH in the cluster.

\section{Summary}

We determine the orbital parameters and the companion mass of PSR B1820-30A using the measured higher-order rotational frequency derivatives through pulsar timing. Our results show that the pulsar is in either a high-eccentricity larger orbit $(e \gtrsim 0.9)$ around the central IMBH or in a low-eccentricity smaller orbit $(0.33 \lesssim e \lesssim 0.4)$ with a low-mass companion of mass $\approx(0.5-4.9) \mathrm{M}_{\odot}$ (i.e. a main-sequence or slightly evolved cluster star, white dwarf, neutron star or stellar mass BH). However, the properties and the measurements of the cluster and the other sources near the centre are consistent 


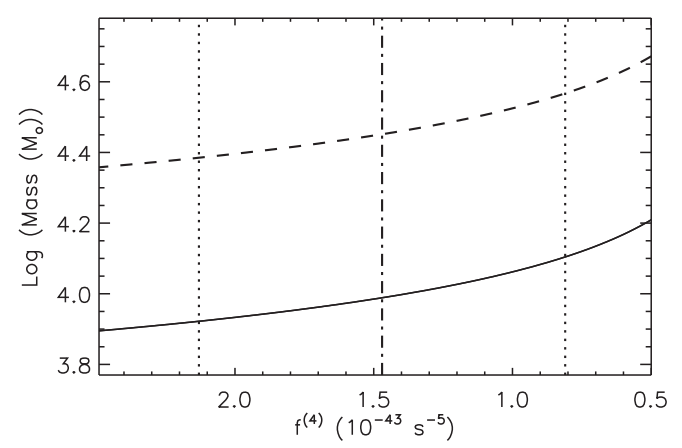

Figure 2. Companion mass of the binary system. The vertical dot-dashed and dotted lines represent the timing measurement of $f^{(4)}$ with its uncertainty. The minimum (solid) and the maximum (dashed) estimates of the mass are obtained based on the orbital inclination $90^{\circ}$ and $44^{\circ}$, respectively. The lower limit of the inclination angle is determined from the maximum measured projected separation of $0.042 \mathrm{pc}$.

with a central IMBH (see Peuten et al. 2014), and thus PSR B1820-30A is highly likely orbiting around a central IMBH, providing larger orbits are more probable. Regardless of the contribution of the pulsar intrinsic spin-down to the observed value shown in timing measurements, we deduce that NGC 6624 contains an IMBH with a mass of $>7500 \mathrm{M}_{\odot}$ at the centre and that the pulsar orbits around it. This is the first evidence and mass constraint of a central $\mathrm{BH}$ in a globular cluster made using the timing measurements of pulsars directly combined with orbital dynamics. The full description of the analysis is given in Perera et al. $(2017 \mathrm{a}, \mathrm{b})$.

Recently, Gieles et al. (2017) reported that the observed period derivatives of PSR B1820-30A can be explained due to passing stars without a central IMBH. However, their simulation showed a concentration of dark remnants in the centre. We note that a detailed analysis is needed to investigate the stability of these period derivative results. We also note that a significant $f^{(5)}$ measurement in the future will further help to strengthen the orbital dynamics of the system.

\section{References}

Biggs J. D., Bailes M., Lyne A. G., Goss W. M., \& Fruchter A. S. 1994, MNRAS, 267, 125

Canizares C. R., Grindlay J. E., Hiltner W. A., Liller W., \& McClintock J. E. 1978, ApJ, 224, 39

Chandler A. M 2003, PhD thesis, California Inst. Technol.

Gieles M., Balbinot E., Yaaqib R., Henault-Brunet V., Zocchi A., Peuten M., \& Jonker P. G. 2017, ArXiv e-prints, 1709.06874

Harris W. E. 1996, AJ, 112, 1487

Joshi K. J. \& Rasio F. A. 1997, ApJ, 479, 948

Kuulkers E., den Hartog P. R., in 't Zand J. J. M., Verbunt F. W. M., Harris W. E., \& Cocchi M. 2003, $A \mathscr{E} A, 399,663$

Lynch R. S., Freire P. C. C., Ransom S. M., \& Jacoby B. A. 2012, ApJ, 745, 109

Manchester R. N., Hobbs G. B., Teoh A., \& Hobbs M. 2005, AJ, 129, 1993

Perera B. B. P., Stappers B. W., Lyne A. G., Bassa C. G., Cognard I., Guillemot L., Kramer M., Theureau G., \& Desvignes G. 2017a, MNRAS, 468, 2114

Perera B. B. P., Stappers B. W., Lyne A. G., Bassa C. G., Cognard I., Guillemot L., Kramer M., Theureau G., \& Desvignes G. 2017b, MNRAS, 471, 1258

Peuten M., Brockamp M., Küpper A. H. W., \& Kroupa P. 2014, ApJ, 795, 116

Portegies Zwart S. F., Baumgardt H., Hut P., Makino J., \& McMillan S. L. W. 2004, Nature, 428,724 